\title{
A 3D Search for the Interplay between AGN and Star Formation in Galaxies
}

\author{
Marsha Wolf ${ }^{1}$, Eric Hooper ${ }^{1}$, Ryan Sanders ${ }^{2}$ and Charles Liu ${ }^{3}$ \\ ${ }^{1}$ Department of Astronomy, University of Wisconsin, Madison, WI 53706, USA \\ email: mwolf@astro.wisc.edu \\ ${ }^{2}$ UCLA Division of Astronomy \& Astrophysics, Los Angeles, CA 90095-1547, USA \\ ${ }^{3}$ Department of Engineering Science and Physics, CUNY CSI, Staten Island, NY 10314, USA
}

\begin{abstract}
Integral field spectroscopy and radio interferometry are very powerful tools for studying the interplay between AGN and star formation (SF) in galaxies. We introduce a sample of SDSS galaxies with selection criteria designed to maximize our chances of catching both processes in action. The galaxies are post-starburst, potentially contain radio AGN, and are allowed, but not required, to have ongoing star formation. The resulting sample includes objects classified as traditional post-starbursts and ones that would have been classified as Seyferts based on their emission line properties alone. The systems span a range of merger phases from initial interaction to fully merged, providing snapshots throughout the entire sequence. We are compiling a multi-wavelength data set, including spatially resolved optical spectra from IFUs on WIYN and continuum radio maps from the VLA and GMRT. Here we present initial results on J0754+1648, an interacting system with a post-starburst region near a radio AGN surrounded by highly ionized gas. This object may be an example of SF truncated by AGN feedback.
\end{abstract}

Keywords. galaxies: interaction - galaxies: evolution - galaxies: active

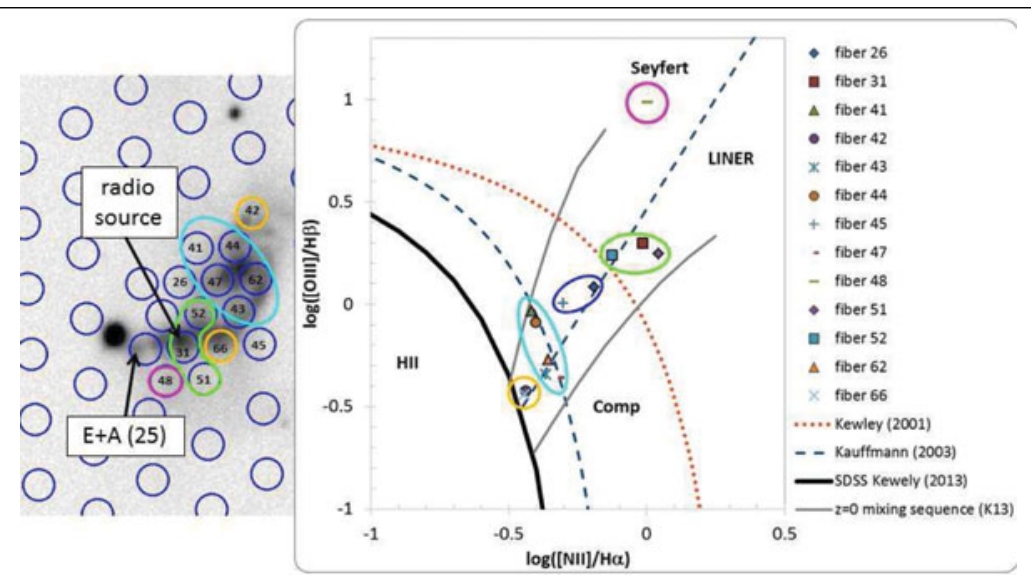

Figure 1. Layout of the SparsePak IFU (Bershady+, 2004, PASP, 116, 565) on J0754+1648 is on the left. Colored outlines mark spatial regions on the object and their corresponding locations on the BPT plot on the right (Kauffmann+, 2003, MNRAS, 346, 1055; Kewley+, 2001, ApJ, 556, 121; Kewley+, 2013, ApJ, 774, 100). The heavy black line is the mean SDSS abundance sequence and solid gray lines bound the starburst-AGN mixing sequence (Kewley+ 2013). The upper nucleus of the system, near fiber 44 , is forming stars at a rate of $4 \mathrm{M}_{\text {sun }} \mathrm{yr}^{-1}$ (Sanders+, 2012, BAAS, 246.07). Fibers in this area (cyan) lie in the SF region of the BPT near the composite line. Fibers $26 \& 45$ that are spatially between the SF area and the AGN lie in the composite region of the BPT (blue). Fiber 31 contains the radio source and lies in the AGN/LINER region of the BPT (green). Fiber 48 has strong [OIII] emission, likely ionized by the AGN, and is fully in the Seyfert BPT region (magenta). The post-starburst (fiber 25) has a mean light-weighted age of $540 \mathrm{Myr}$ and is in close proximity to the AGN and the ionized gas. 